\title{
Nutrient intake disparities in the US: modeling the effect of food substitutions
}

\author{
Zach Conrad ${ }^{1 *}$ (D), LuAnn K. Johnson', James N. Roemmich', WenYen Juan² and Lisa Jahns ${ }^{1}$
}

\begin{abstract}
Background: Diet quality among federal food assistance program participants remains low, and little research has assessed the diet quality of food insecure non-participants. Further research is needed to assess the extent to which food substitutions can improve the nutritional status of these vulnerable populations. Substituting egg dishes for other commonly consumed dishes at certain eating occasions may be an effective strategy for improving the daily nutrient intake among these groups. Eggs are rich in many important nutrients, and are low-cost and part of a wide range of cultural food menus, which are important considerations for low-income and ethnically diverse populations. To help guide the focus of targeted nutrition interventions and education campaigns for vulnerable populations, the present work begins by 1) estimating the prevalence of nutrient inadequacy among these groups, and then models the effect of consuming egg dishes instead of commonly consumed dishes at each eating occasion on 2) the prevalence of nutrient inadequacy, and 3) the mean intake of nutrients.

Methods: Dietary data from 34,741 adults $\geq 20$ y were acquired from the National Health and Nutrition Examination Survey, 2001-2014. Diet pattern modeling was used to substitute commonly consumed egg dishes for commonly consumed main dishes at breakfast, lunch, and dinner. National Cancer Institute usual intake methods were used to estimate the prevalence of inadequate intake of 31 nutrients pre- and post-substitution, and a novel index was used to estimate change in intake of all nutrients collectively.

Results: Substituting eggs for commonly consumed main dishes at lunch or dinner did not change total daily nutrient intake for each group $(P>0.05)$, but decreased the prevalence of vitamin $D$ inadequacy by $1-4$ percentage points $(P<0.01)$. Substituting eggs for commonly consumed foods at breakfast increased the prevalence of folate inadequacy by $8-12$ percentage points among each group $(P<0.01)$.

Conclusions: When making food substitutions to increase nutrient intake, eating occasion should be an important consideration. Further research is needed to better understand how food substitutions affect diet costs, which may be an important driver of food purchasing decisions among low income individuals with limited food budgets.

Keywords: Diet quality, Nutrient intake, Food security, Food assistance, Diet model
\end{abstract}

\section{Background}

Federal food assistance programs in the US have expanded in scale and number over the past century, and are now a cornerstone of the national nutrition agenda. These programs represent essential tools to reduce food insecurity and improve the diet quality of low-income individuals and households across the country.

\footnotetext{
* Correspondence: zach.conrad@ars.usda.gov

${ }^{1}$ Grand Forks Human Nutrition Research Center, US Department of Agriculture, Agricultural Research Service, 2420 2nd Ave. N, Grand Forks, ND 58203, USA

Full list of author information is available at the end of the article
}

The largest of these programs, in federal financial outlays and the number of individuals participating, are the Supplemental Nutrition Assistance Program (SNAP) and Special Supplemental Nutrition Program for Women, Infants, and Children (WIC). SNAP, formerly known as the "Food Stamp Program", provides financial benefits to eligible households to purchase foods for at-home consumption. Eligibility is determined primarily by household income at or below $130 \%$ of the poverty level [1]. Over 45 million individuals participated in SNAP in 2016, [2] with annual program costs exceeding $\$ 80$ billion [3]. In contrast to SNAP, WIC provides financial

(C) The Author(s). 2018 Open Access This article is distributed under the terms of the Creative Commons Attribution 4.0 International License (http://creativecommons.org/licenses/by/4.0/), which permits unrestricted use, distribution, and 
benefits to eligible individuals to purchase a select suite of approved foods [4]. Eligibility is determined by a categorical requirement (pregnant, post-partum, or breastfeeding women; infants; and children), income requirement (statespecific income-to-poverty ratio threshold), and nutrition risk requirement (individuals deemed to be at risk of nutrition-related poor health outcomes). Approximately 9.5 million individuals participated in WIC in 2016, [4] with a program cost of $\$ 6.6$ billion [3].

Low diet quality is the leading cause of mortality in the US [5]. Income is an important moderator of diet quality, with lower income individuals exhibiting lower quality diets than their higher income counterparts $[6$, 7]. SNAP and WIC participants, in particular, generally exhibit low quality diets $[8,9]$ and high rates of cardiometabolic mortality [10]. Yet little research has assessed the diet quality of food insecure individuals who are not participating in either SNAP or WIC. Further efforts are needed to address this important research gap and to assess interventions to improve the nutritional status of these vulnerable populations.

Evidence suggests that moderate diet and lifestyle changes can be more easily adopted and sustained than more drastic changes, and can ultimately elicit meaningful health improvements [11-13]. Following this principle, one approach to improving diet quality is to counsel individuals to make healthier food choices one meal at a time. Several studies have found that the greatest amount of calories is consumed at dinner, yet the intake of micronutrients at each eating occasion was not examined [14-16]. Others have examined the micronutrient contribution of individual foods to daily nutrient intakes, such as beef, [17] dairy, [18-20] breakfast cereal, [21] orange juice, [22] potatoes, [23] avocados, [24] and canned fruits and vegetables, [25] yet evidence is lacking on how specific food choices at each eating occasion (i.e. breakfast, lunch, and dinner) contribute to daily nutrient intake.

Substituting egg dishes for other commonly consumed dishes at certain eating occasions may be an effective strategy for improving the daily nutrient intake among vulnerable populations. Eggs are rich in many important nutrients, [26-28] increase the bioavailability of coconsumed carotenoids [29] and vitamin E, [30] and promote positive health outcomes at all ages and all stages of life [30, 31]. In a recent study, pregnant egg consumers were much more likely to meet daily choline intake recommendations than pregnant non-egg consumers [32]. And eggs are low-cost and part of a wide range of cultural food menus, [31] which are important considerations for low-income and ethnically diverse populations.

To help guide the focus of targeted nutrition interventions and education campaigns for individuals participating in federal food assistance programs and those with low food security, the present work begins by 1) estimating the prevalence of nutrient inadequacy among these groups, and then models the effect of consuming egg dishes instead of commonly consumed dishes at each eating occasion on 2) the prevalence of nutrient inadequacy, and 3) the mean intake of nutrients.

\section{Methods \\ Dietary data}

Data on individual-level food and nutrient intake, SNAP and WIC participation, food security status, and other characteristics were acquired from the National Health and Nutrition Examination Survey (NHANES), waves 20012002, 2003-2004, 2005-2006, 2007-2008, 2009-2010, 2011-2012, and 2013-2014 [33]. NHANES is a continuous, cross-sectional survey that collects data on demography and health behaviors from a sample of $\sim 5000$ individuals per year, and data are released in two-year cycles. Data on food and nutrient intake were acquired from What We Eat In America (WWEIA), the dietary component of NHANES. Individuals complete a $24-\mathrm{h}$ recall $(24 \mathrm{HR})$ administered by a trained interviewer using United States Department of Agriculture's (USDA) Automated Multiple Pass Method, [34] and a subset of the study population $(\sim 80 \%)$ completes a subsequent $24 \mathrm{HR}$ by telephone on a non-consecutive day. Food security status was assessed by trained NHANES staff using the US Food Security Module [35].

\section{Study populations}

All individuals $20+\mathrm{y}$ with dietary recalls that were considered reliable by trained NHANES staff were included in this study $(n=34,741)$. Respondents were categorized as SNAP participants, WIC participants, food insecure non-participants, and food secure non-participants. SNAP participants were identified as individuals who reported that someone in their household was participating in SNAP at the time of the survey $(n=4020)$, and WIC participants were identified as individuals who reported that they were participating in WIC at the time of the survey $(n=636)$. Individuals who reported participating in SNAP and WIC at the time of the survey $(n=$ 209) were categorized as WIC participants in order to maintain the relative homogeneity of this group compared to the SNAP group (due to the more restrictive eligibility requirements of WIC). Individuals who did not report participating in SNAP or WIC but reported low or very low food security were categorized as food insecure non-participants $(n=3631)$, and individuals who did not report participating in SNAP or WIC and reported marginal or full food security were categorized as food secure non-participants $(n=26,454)$.

\section{Identifying main dishes at each eating occasion}

For each individual food reported consumed in WWEIA 2001-2014, the eating occasion and time of day each 
food was consumed was reported in either English or Spanish. Foods reported consumed at breakfast, lunch, or dinner were grouped into main dish categories primarily according to the categorization scheme used in the Food and Nutrient Database for Dietary Studies (FNDDS; Additional file 1: Table S1) [36]. A total of 17 main dish categories were identified: beef dishes, pork dishes, bacon, poultry dishes, other meat dishes, seafood dishes, sandwiches, sausages, soups, breads, sweet baked goods, pancakes and waffles, oatmeal, breakfast cereal, pasta, Mexican dishes, and pizzas and calzones. Additionally, six egg dishes were identified: whole eggs, scrambled eggs and omelets, egg sandwiches, egg soups, frozen egg dishes, and quiches. A composite nutrient profile was created for each main dish and each egg dish by averaging the nutrient content per gram weight of each of the foods in each dish category.

\section{Diet modeling}

A diet model was constructed to estimate the effects on daily nutrient intake if egg dishes were substituted for commonly consumed main dishes at each eating occasion (i.e. breakfast, lunch, and dinner). Commonly consumed dishes at each eating occasion among each group (i.e. SNAP participants, WIC participants, food insecure non-participants, and food secure non-participants) were identified by frequency of reported consumption (i.e. percent of individuals reporting consumption of each dish). The diet model replaced the most commonly consumed main dish with the most commonly consumed egg dish at each eating occasion for each individual in each group, on a gram weight basis. For example, if the most commonly consumed main dish among SNAP participants at breakfast was bread, and the most commonly consumed egg dish among SNAP participants at breakfast was scrambled eggs, then for each SNAP participant the amount of bread consumed (in grams) was replaced with the same gram weight of scrambled eggs.

\section{Total nutrient index}

A total nutrient index was developed to provide a summary estimate of how much nutrient intake changed (for all nutrients collectively) when egg dishes were consumed instead of the most commonly consumed dishes at each eating occasion. This index is interpreted as the mean percent change in nutrient intake from baseline intake to modeled intakes. The total nutrient index was calculated in two steps: first, by estimating the percent change in intake from baseline for each individual nutrient, and then 2) computing the mean percent change in nutrient intake across all nutrients. Nutrients which Americans are encouraged to limit, including sodium and saturated fat, were reverse scored prior to computing the total nutrient index so that greater values represented lower percent change from baseline. To ensure that more accurately measured nutrient intakes had greater influence on the index, the total nutrient index was weighted by the standard error of each nutrient, so that nutrients with greater standard errors received less weight in the index. The total nutrient index can be expressed as:

$$
\text { Total nutrient index }=\frac{\sum_{i=1}^{N} C_{i} / s e_{i}}{\sum_{i=1}^{N} s e_{i}}
$$

where $C$ is the percent change in the intake of a given nutrient $(i)$ weighted by its standard error (se), and $N$ is the number of nutrients included in the index.

Sensitivity analyses were used to test the sensitivity of the total nutrient index to potentially influential individual nutrients. In these analyses, nutrients with the greatest change from baseline were iteratively removed from the index and compared against the original index.

\section{Statistical analyses}

Daily nutrient intake was estimated at baseline (i.e. reported daily intake) and for three modeled intakes: substituting egg dishes for the most commonly consumed main dishes at breakfast, lunch, and dinner. The percent of individuals in each group not meeting age-and sex-specific daily intake recommendations, as well as the mean daily intake of each nutrient, were estimated using the National Cancer Institute's (NCI) usual intake methodology [37]. The NCI method uses information from two 24HRs to estimate within-person variation using the SAS macros MIXTRAN and DISTRIB. MIXTRAN uses logistic regression with a person-specific random effect to estimate the probability of consumption, accounting for the day of intake and whether it was a weekday or weekend; DISTRIB estimates daily intake; and the results from both macros are linked with a correlated person-specific random effects model.

The percent of individuals not meeting daily intake recommendations was estimated for the 16 nutrients with an Estimated Average Requirement (EAR; vitamins $\mathrm{A}, \mathrm{B}_{6}, \mathrm{~B}_{12}, \mathrm{C}, \mathrm{D}, \mathrm{E}$; thiamin; riboflavin; niacin; folate; calcium; iron; magnesium; and zinc), an Upper Limit (UL; sodium), or a Dietary Guidelines for Americans 2015-2020 (DGA) recommendation (saturated fatty acids; Additional file 2: Table S2). Mean nutrient intakes were estimated for all nutrients, additionally including 12 nutrients without a recommended intake amount: fiber, eicosapentanoic acid, docosahexaenoic acid, $\alpha$ linolenic acid, vitamin $\mathrm{K}$, choline, potassium, $\alpha$-carotene, $\beta$-carotene, $\beta$-cryptoxanthin, lutein and zeaxanthin, and lycopene. Mean intakes were also estimated for total protein, carbohydrate, and fat. The balanced repeated replication method was used to estimate standard errors while accounting for the complex sampling design of NHANES. 
All analyses were adjusted for age (20-30, 31-50, 5170, 71+), gender, race-ethnicity (non-Hispanic white, non-Hispanic black, Mexican-American), education (less than high school, high school or equivalent, some college, college graduate), marital status (single and never married; married or living with a partner; widowed, divorced, or separated), household size (1, 2, 3-4, 5+), currently participating in a health insurance plan (yes, no), body mass index (less than 18.5, 18.5-24.9, 25-29.9, $30+$ ), income-to-poverty ratio (less than $0.5,0.51-1.0,1$. 01-1.3, greater than 1.3), and whether the dietary recall was completed on a weekday or weekend. Differences in baseline nutrient intake across groups, and differences between baseline intake and each of the modeled intakes within each group were tested using z-scores, with significance at $P<0.05$ with Bonferroni adjustment for multiple comparisons. Stata 14 was used for data management and SAS 9.4 was used for all analyses. This study was deemed exempt by the University of North Dakota Institutional Review Board.

\section{Results}

\section{Sociodemographic characteristics}

WIC participants were younger (26.3 y, 95\% CI: $25.6-26.9$ y) than all other groups (Table 1), and food secure nonparticipants were older $(48.0 \mathrm{y}, 47.5-48.5$ y). All WIC participants were female, and between 50 and $60 \%$ of food insecure non-participants, SNAP participants, and food secure non-participants were female. Most individuals in each group were non-Hispanic white, with the greatest proportion in the food secure non-participant group $(82.7 \%, 80.7-84.6 \%)$. All groups had a similar distribution of educational attainment except for food secure non-participants, which had higher educational attainment compared to the other groups. Most individuals in each group were married or living with a partner, with the greatest proportion in the WIC participant group $(67.8 \%, 61.9-73.2 \%)$ and food secure non-participant group (65.9\%, 64.6-67.1\%). WIC participants reported the greatest household size (4.3, 4.2-4.5), and food secure non-participants reported the lowest household size $(2.9,2.8-2.9)$. Food secure non-participants reported the highest prevalence (84.9\%, 83.9-85.8\%) of currently participating in a health insurance plan, followed by WIC participants $(74.6 \%, 69.0-79.4 \%)$, SNAP participants $(67.3 \%, 64.7-70.0 \%)$, and food insecure non-participants $(58.0 \%, 55.3-60.6 \%)$. Overweight and obese individuals accounted for $67-76 \%$ of each group, yet food secure non-participants had the highest proportion of normal weight individuals (31.2\%, 30.2-32.2\%). Most $(87.2 \%, 86.2-88.1 \%)$ food secure non-participants had an income-to-poverty ratio greater than 1.3, compared with $46.9 \%$ (43.9-50.0\%) of food insecure non-participants, $35.5 \%(30.0-41.4 \%)$ of WIC participants, and $24.7 \%(21.7-28.1 \%)$ of SNAP participants.

Most commonly consumed dishes at each eating occasion Among each group, the most commonly consumed main dishes at breakfast, lunch, and dinner were breakfast cereal, sandwiches (including hotdogs and sausages), and poultry dishes (including mixed dishes with poultry and vegetables), respectively (Additional file 3: Table S3). Whole eggs and scrambled eggs were the most commonly consumed egg dishes at breakfast, lunch, and dinner among each group (Additional file 4: Table S4). Mean consumption amounts and nutrient profiles are presented in Additional file 5: Table S5 and Additional file 6: Table S6, respectively.

\section{Reported nutrient intake}

Table 2 displays reported daily nutrient intakes among each group, with food insecure non-participants as the reference group. The percent not meeting recommendations for vitamin $\mathrm{C}$ and calcium was lower, and the percent not meeting recommendations for vitamin $\mathrm{E}$ was higher, among WIC participants compared to food insecure nonparticipants $(P<0.001$ for vitamin $C$, and $P<0.01$ for vitamin $\mathrm{E}$ and calcium). WIC participants also had higher mean intakes of energy $(P=0.023)$, protein $(P=0.009)$ , and carbohydrates $(P=0.007)$ compared to food insecure non-participants. The percent not meeting recommendations for vitamin D among food insecure non-participants $(98.0 \%, 97.8-98.2 \%)$ was higher than SNAP participants $(96.0 \%, 95.8-96.2 \% ; P<0.001)$; and the percent not meeting recommendations for folate (30.9\%, 28.4-33.5\%) and zinc (47.0\%, 44.7-49.3\%) among food insecure non-participants was lower than SNAP participants (folate: 35.7\%, 33.2-38.1\%; $P<0.05$ and zinc: $52.4 \%, 49.7-55.2 \% ; P<0.01)$. Compared to food insecure non-participants, the percent not meeting recommendations was lower among food secure non-participants for all nutrients $(P<0.01$ or $P<0.001$ for all comparisons) except for saturated fatty acids (higher, $P<0.01$ ), sodium (higher, $P<0.001$ ), niacin (no difference, $P=0.251$ ), and folate (no difference, $P=0.036$ ).

No difference in mean intake was observed between food insecure non-participants and WIC participants (Table 2$)$ for total fatty acids $(P=0.045)$, fiber $(P=0.808)$, $\alpha$-linolenic acid $(P=0.776)$, vitamin $\mathrm{K} \quad(P=0.373)$, choline $(P=0.033)$, potassium $(P=0.557), \alpha$-carotene $(P=0.455), \quad \beta$-carotene $(P=0.846), \quad \beta$-cryptoxanthin $(P=0.109)$, lutein and zeaxanthin $(P=0.557)$, and lycopene $(P=0.620)$. Compared to food insecure nonparticipants, SNAP participants had lower mean intake of fiber and lycopene $(P<0.01$ for both comparisons), and higher mean intake of $\alpha$-carotene $(P<0.001)$ and $\beta$-carotene $(P<0.01)$. Food secure non-participants had lower mean 
Table 1 Sociodemographic characteristics of participants in federal food assistance programs, food insecure individuals, and other individuals 2001-2014 $(n=34,741)$

\begin{tabular}{|c|c|c|c|c|c|c|c|c|c|}
\hline \multirow[t]{2}{*}{ Characteristic } & $n$ & \multicolumn{2}{|c|}{$\begin{array}{l}\text { Food insecure, non-participants } \\
(\mathrm{n}=3631)\end{array}$} & \multicolumn{2}{|c|}{$\begin{array}{l}\text { WIC participants } \\
(n=636)\end{array}$} & \multicolumn{2}{|c|}{$\begin{array}{l}\text { SNAP participants } \\
(n=4020)\end{array}$} & \multicolumn{2}{|c|}{$\begin{array}{l}\text { Food secure, non-participants } \\
(n=26,454)\end{array}$} \\
\hline & & & & & $\%(95 \% \mathrm{Cl})^{\mathrm{a}}$ & & & & \\
\hline Age (y) & 34,741 & 41.5 & $(40.5-42.4)$ & 26.3 & $(25.6-26.9)$ & 42.9 & $(42.1-43.7)$ & 48.0 & $(47.5-48.5)$ \\
\hline Female & 34,741 & 50.0 & $(47.8-51.4)$ & 100.0 & (NA) & 59.0 & $(57.1-60.9)$ & 50.7 & $(50.1-51.3)$ \\
\hline Race-ethnicity & 29,696 & & & & & & & & \\
\hline Non-Hispanic white & & 56.9 & $(52.0-61.6)$ & 43.0 & $(35.4-50.8)$ & 56.7 & $(50.7-62.4)$ & 82.7 & $(80.7-84.6)$ \\
\hline Non-Hispanic black & & 20.3 & $(17.5-23.5)$ & 28.3 & $(22.6-34.9)$ & 30.6 & $(26.3-25.4)$ & 10.0 & $(8.8-11.3)$ \\
\hline Mexican American & & 22.8 & $(19.3-26.8)$ & 28.7 & $(22.8-35.4)$ & 12.7 & $(9.7-16.4)$ & 7.3 & $(6.1-8.6)$ \\
\hline Education & 34,704 & & & & & & & & \\
\hline Less than high school & & 33.9 & $(31.1-36.7)$ & 34.0 & $(28.8-40.0)$ & 39.5 & $(37.0-42.1)$ & 13.6 & $(12.6-14.7)$ \\
\hline High school or equivalent & & 27.0 & $(24.8-29.2)$ & 34.1 & $(26.7-36.5)$ & 28.4 & $(26.1-31.0)$ & 22.9 & $(21.8-24.0)$ \\
\hline Some college & & 31.4 & $(28.8-34.2)$ & 29.0 & $(24.2-34.2)$ & 27.1 & $(25.1-29.1)$ & 31.8 & $(30.8-32.7)$ \\
\hline College graduate & & 7.7 & $(6.4-9.3)$ & 5.6 & $(3.7-8.5)$ & 5.0 & $(3.9-6.3)$ & 31.7 & $(30.1-33.4)$ \\
\hline Marital status & 34,723 & & & & & & & & \\
\hline Single, never married & & 25.0 & $(22.3-27.9)$ & 26.6 & $(21.6-32.1)$ & 29.5 & $(26.8-32.4)$ & 16.7 & $(15.5-18.0)$ \\
\hline Married or living with partner & & 50.5 & $(47.6-53.4)$ & 67.8 & $(61.9-73.2)$ & 39.7 & $(37.1-42.5)$ & 65.9 & $(64.6-67.1)$ \\
\hline $\begin{array}{l}\text { Widowed, divorced, or } \\
\text { separated }\end{array}$ & & 24.5 & $(22.4-26.8)$ & 5.7 & $(3.5-9.0)$ & 30.7 & $(28.3-33.2)$ & 17.4 & $(16.7-18.1)$ \\
\hline Household size (n) & 34,741 & 3.3 & $(3.2-3.4)$ & 4.3 & $(4.2-4.5)$ & 3.6 & $(3.46-3.78)$ & 2.9 & $(2.8-2.9)$ \\
\hline Health insurance ${ }^{b}$ & 34,620 & 58.0 & $(55.3-60.6)$ & 74.6 & $(69.0-79.4)$ & 67.3 & $(64.7-70.0)$ & 84.9 & (83.9-85.8) \\
\hline Weight status & 34,066 & & & & & & & & \\
\hline Underweight & & 2.6 & $(1.9-3.5)$ & 0.9 & $(0.3-2.5)$ & 2.7 & $(2.1-3.6)$ & 1.5 & $(1.3-1.7)$ \\
\hline Normal weight & & 28.5 & $(26.2-31.0)$ & 22.9 & $(18.3-28.2)$ & 25.5 & $(23.5-27.6)$ & 31.2 & $(30.2-32.2)$ \\
\hline Overweight & & 30.0 & $(27.7-32.4)$ & 32.2 & $(26.7-38.3)$ & 27.7 & $(25.7-29.8)$ & 34.6 & $(33.6-35.6)$ \\
\hline Obese & & 38.9 & $(36.4-41.5)$ & 44.0 & $(38.6-49.7)$ & 44.1 & $(41.3-46.8)$ & 32.7 & $(31.6-33.8)$ \\
\hline Income-to-poverty ratio & 32,247 & & & & & & & & \\
\hline Less than or equal to 0.50 & & 11.3 & $(9.5-13.3)$ & 19.9 & $(15.2-25.5)$ & 21.1 & $(18.9-23.5)$ & 2.3 & $(1.9-2.7)$ \\
\hline 0.51 to 1.00 & & 22.0 & $(19.6-24.6)$ & 30.9 & $(26.2-36.1)$ & 40.3 & $(37.0-43.7)$ & 5.1 & $(4.7-5.6)$ \\
\hline 1.01 to 1.30 & & 19.9 & $(17.4-22.5)$ & 13.7 & $(10.3-18.1)$ & 13.8 & $(12.5-15.3)$ & 5.4 & $(4.9-6.0)$ \\
\hline$>1.30$ & & 46.9 & $(43.9-50.0)$ & 35.5 & $(30.0-41.4)$ & 24.7 & $(21.7-28.1)$ & 87.2 & $(86.2-88.1)$ \\
\hline
\end{tabular}

${ }^{a}$ Does not apply to age or household size

${ }^{b}$ Participated in health insurance plan at time of survey

Some SNAP participants may have income-to-poverty ratios greater than 1.3 because income test are the primary, but not the only, criterion of program eligibility. For example, Income tests are relaxed if all family members participate in Temporary Assistance for Needy Families (TANF) or receive Supplemental Security Income (SSI). Additionally, individuals move in and out of SNAP on a monthly basis whereas income data were collected on an annual basis

intake of potassium, and higher mean intake of protein, fiber, $\alpha$-linolenic acid, vitamin $K$, choline, $\alpha$-carotene, $\beta$-carotene, $\beta$-cryptoxanthin, and lutein and zeaxanthin compared to food insecure non-participants $(P<0.001$ for all comparisons except protein: $P=0.012$ ).

\section{Modeled nutrient intake: substituting eggs as the main dish}

Tables 3, 4, 5 and 6 display the results of models that substituted the most commonly consumed egg dish for the most commonly consumed main dish at each eating occasion among food insecure non-participants (Table 3), WIC participants (Table 4), SNAP participants (Table 5), and food secure non-participants (Table 6).
Among food insecure non-participants, no changes in the percent not meeting recommended nutrient intakes were observed when eggs were substituted at breakfast (Table 3). At lunch, substituting eggs decreased the percent not meeting daily recommendations for saturated fatty acids $(P<0.001)$, vitamin $\mathrm{D}(P<0.01)$, and magnesium $(P<$ $0.001)$. When eggs were substituted as the main dish at dinner, the percent not meeting recommendations decreased for vitamin $\mathrm{A}(P<0.01)$ and vitamin $\mathrm{D}(P<0.01)$. Among WIC participants (Table 4), substituting eggs at breakfast increased the percent not meeting folate recommendations $(P<0.01)$; at lunch, the percent not meeting recommendations decreased for saturated fatty acids $(P<0.01)$ and magnesium $(P<0.001)$, and mean intake of choline increased 
Table 2 Reported daily nutrient intakes among federal food assistance program participants, food insecure non-participants, and food secure non-participants, 2001-2014 ( $n=34,741)$

\begin{tabular}{|c|c|c|c|c|c|c|c|c|}
\hline \multirow{3}{*}{$\begin{array}{l}\text { Nutrient } \\
\text { Saturated fatty acids }{ }^{c}\end{array}$} & \multicolumn{2}{|c|}{$\begin{array}{l}\text { Food insecure non-participants }{ }^{a} \\
(n=3631)\end{array}$} & \multicolumn{2}{|c|}{ WIC participants $(n=636)$} & \multicolumn{2}{|c|}{ SNAP participants $(n=4020)$} & \multicolumn{2}{|c|}{ Food secure non-participants $(26,454)$} \\
\hline & \multicolumn{8}{|c|}{ Percent not meeting recommendations $(95 \% \mathrm{Cl})^{\mathrm{d}}$} \\
\hline & 55.3 & $(52.1-58.5)$ & 57.5 & $(50.4-64.7)$ & 58.9 & $(55.5-62.2)$ & 62.1 & $(60.6-63.6)^{* *}$ \\
\hline Vitamin $A^{d}$ & 67.5 & $(64.7-70.2)$ & 60.9 & $(53.3-68.6)$ & 66.4 & $(62.8-70.0)$ & 46.9 & $(45.4-48.4)^{* * *}$ \\
\hline Vitamin C & 58.7 & $(55.9-61.4)$ & 47.3 & $(39.2-55.3)^{* * * *}$ & 60.3 & $(57.4-55.3)$ & 45.9 & $(44.3-47.5)^{* * *}$ \\
\hline Vitamin D & 98.0 & $(97.8-98.2)$ & 96.0 & $(94.0-98.0)$ & 96.0 & $(95.8-96.2)^{* * *}$ & 95.0 & $(94.8-95.2)^{* * *}$ \\
\hline Vitamin $\mathrm{E}$ & 94.9 & $(93.9-95.8)$ & 97.3 & $(95.9-98.6)^{* *}$ & 95.1 & $(94.1-96.1)$ & 88.1 & $(87.0-89.2)^{* * *}$ \\
\hline Thiamin & 12.0 & $(10.5-13.6)$ & 15.8 & $(12.6-18.9)$ & 11.6 & $(10.2-13.0)$ & 9.5 & $(8.8-10.2)^{* * *}$ \\
\hline Riboflavin & 12.5 & $(10.9-14.0)$ & 12.7 & $(9.8-15.6)$ & 11.6 & $(10.2-13.0)$ & 9.7 & $(9.0-10.4)^{* * *}$ \\
\hline Niacin & 10.2 & $(8.8-11.6)$ & 9.6 & $(6.9-12.3)$ & 9.6 & $(8.2-10.9)$ & 9.3 & $(8.5-10.0)$ \\
\hline Vitamin $B_{6}$ & 22.6 & $(20.5-24.6)$ & 25.7 & $(20.6-30.9)$ & 23.8 & $(21.9-25.8)$ & 18.8 & $(17.6-19.9)^{* * *}$ \\
\hline Folate $^{e}$ & 30.9 & $(28.4-33.5)$ & 27.3 & $(22.3-32.2)$ & 35.7 & $(33.2-38.1)^{*}$ & 27.9 & $(26.8-29.0)$ \\
\hline Vitamin $B_{12}$ & 13.6 & $(12.0-15.3)$ & 11.3 & $(8.7-13.9)$ & 12.3 & $(10.7-13.9)$ & 10.8 & $(10.0-11.6)^{* *}$ \\
\hline Calcium & 54.7 & $(52.0-57.4)$ & 44.6 & $(37.8-51.4)^{* *}$ & 55.5 & $(52.7-58.2)$ & 47.4 & $(46.2-48.6)^{* * *}$ \\
\hline Iron & 15.7 & $(14.1-17.4)$ & 62.6 & $(50.6-74.6)^{* * *}$ & 17.2 & $(15.4-19)$ & 12.7 & $(11.9-13.5)^{* * *}$ \\
\hline Magnesium & 83.1 & $(81.3-84.9)$ & 86.9 & $(83.5-90.4)$ & 85.8 & $(84.1-87.6)$ & 72.1 & $(70.9-73.3)^{* * *}$ \\
\hline Sodium $^{f}$ & 78.7 & $(76.9-80.5)$ & 81.4 & $(76.6-86.2)$ & 76.5 & $(74.5-78.5)$ & 81.4 & $(80.5-82.3)^{* * *}$ \\
\hline \multirow[t]{2}{*}{ Zinc } & 47.0 & $(44.7-49.3)$ & 44.2 & $(38.5-49.9)$ & 52.4 & $(49.7-55.2)^{* *}$ & 39.4 & $(38.2-40.6)^{* *}$ \\
\hline & \multicolumn{8}{|c|}{ Mean intake $(95 \% \mathrm{Cl})$} \\
\hline Energy (kcal) & 2171 & $(2119-2224)$ & 2309 & $(2202-2416)^{*}$ & 2192 & $(2144-2239)$ & 2176 & $(2159-2192)$ \\
\hline Protein, total (g) & 81 & $(79-83)$ & 88 & $(83-92)^{*}$ & 81 & $(80-83)$ & 84 & $(83-84)^{*}$ \\
\hline Carbohydrate, total (g) & 267 & $(260-274)$ & 289 & $(275-304)^{*}$ & 270 & $(263-277)$ & 262 & $(260-264)$ \\
\hline Fatty acids, total (g) & 81 & $(78-83)$ & 87 & $(81-92)$ & 81 & (79-83) & 83 & $(82-84)$ \\
\hline Fiber $(g)$ & 14 & $(13-14)$ & 14 & $(13-15)$ & 13 & $(12-13)^{* *}$ & 16 & $(16-16)^{* * *}$ \\
\hline Eicosapentaenoic acid (g) & 0.02 & $(0.02-0.02)$ & 0.02 & $(0.02-0.03)$ & 0.02 & $(0.02-0.02)$ & 0.03 & $(0.03-0.03)^{* *}$ \\
\hline Docosahexaenoic acid (g) & 0.07 & $(0.06-0.08)$ & 0.07 & $(0.06-0.08)$ & 0.06 & $(0.06-0.06)^{* *}$ & 0.08 & $(0.08-0.08)^{*}$ \\
\hline a-linolenic acid (g) & 1.40 & $(1.35-1.44)$ & 1.38 & $(1.26-1.5)$ & 1.35 & $(1.31-1.4)$ & 1.53 & $(1.5-1.55)^{* * *}$ \\
\hline Vitamin $\mathrm{K}(\mu \mathrm{g})$ & 76 & $(72-79)$ & 72 & $(65-80)$ & 72 & $(68-76)$ & 100 & $(97-103)^{* * *}$ \\
\hline Choline (mg) & 291 & $(282-300)$ & 269 & $(251-287)$ & 294 & $(285-302)$ & 317 & $(312-322)^{* * *}$ \\
\hline Potassium (mg) & 2586 & $(2555-2618)$ & 2252 & (2113-2392) & 2586 & $(2555-2618)$ & 2222 & $(2159-2286)^{* * *}$ \\
\hline a-carotene $(\mu \mathrm{g})$ & 320 & $(292-349)$ & 352 & $(274-430)$ & 516 & $(488-544)^{* * *}$ & 516 & $(488-544)^{* * *}$ \\
\hline$\beta$-carotene $(\mu \mathrm{g})$ & 1417 & $(1331-1504)$ & 1395 & $(1187-1603)$ & 2136 & $(2054-2218)^{* *}$ & 2136 & $(2054-2218)^{* * *}$ \\
\hline$\beta$-cryptoxanthin $(\mu \mathrm{g})$ & 84 & $(78-90)$ & 102 & $(81-123)$ & 78 & $(73-83)$ & 112 & $(108-116)^{* * *}$ \\
\hline Lutein and zeaxanthin $(\mu \mathrm{g})$ & 998 & $(938-1058)$ & 1040 & $(914-1166)$ & 931 & $(875-987)$ & 1418 & $(1362-1473)^{* * *}$ \\
\hline Lycopene $(\mu \mathrm{g})$ & 7514 & $(6764-8264)$ & 7939 & $(6436-9442)$ & 6184 & $(5627-6742)^{* *}$ & 8218 & (7838-8598) \\
\hline
\end{tabular}

All estimates adjusted for age, gender, race-ethnicity, education, marital status, household size, health insurance, BMl, income-to-poverty ratio, and whether the dietary recall was on a weekday or weekend

${ }^{\text {a }}$ Reference group

${ }^{b}$ Daily nutrient recommendations are Estimated Average Requirements (EAR) unless otherwise specified. National Academy of Sciences, Institute of Medicine. 2006. Dietary reference intakes: The essential guide to nutrient requirements. Jennifer J. Otten, Jennifer Pitzi Hellwig, Linda D. Meyers (eds.). National Academies Press, Washington, DC

${ }^{C}$ Recommendation is less than $10 \%$ of total energy. US Department of Health and Human Services \& US Department of Agriculture. 2015-2020. Dietary Guidelines for Americans 2015-2020. U.S. Government Printing Office, Washington, DC. Available at: http://health.gov/dietaryguidelines/ (verified 29 March 2016)

${ }^{\mathrm{d}}$ Retinol Activity Equivalent (RAE)

'Dietary Folate Equivalent (DFE)

${ }^{6}$ Tolerable upper intake level limit (UL). National Academy of Sciences, Institute of Medicine. 2006. Dietary reference intakes: The essential guide to nutrient

requirements. Jennifer J. Otten, Jennifer Pitzi Hellwig, Linda D. Meyers (eds.). National Academies Press, Washington, DC

*Different than food insecure non-participants at $P<0.017$ (Bonferroni adjusted for multiple comparisons)

**Different than food insecure non-participants at $P<0.003$ (Bonferroni adjusted for multiple comparisons)

***Different than food insecure non-participants at $P<0.0003$ (Bonferroni adjusted for multiple comparisons) 
Table 3 Reported daily nutrient intakes and modeled nutrient intakes if eggs were consumed as the main dish at each eating occasion among food insecure non-participants, 2001-2014 ( $n=3631)$

\begin{tabular}{|c|c|c|c|c|c|c|c|c|}
\hline \multirow[t]{3}{*}{ Nutrient } & \multirow{2}{*}{\multicolumn{2}{|c|}{$\begin{array}{l}\text { Reported daily } \\
\text { intake }^{2,3}\end{array}$}} & \multicolumn{6}{|c|}{ Modeled daily intake ${ }^{a}$} \\
\hline & & & \multicolumn{2}{|c|}{$\begin{array}{l}\text { Eggs consumed as main dish at } \\
\text { breakfast }\end{array}$} & \multicolumn{2}{|c|}{$\begin{array}{l}\text { Eggs consumed as main dish at } \\
\text { lunch }\end{array}$} & \multicolumn{2}{|c|}{$\begin{array}{l}\text { Eggs consumed as main dish at } \\
\text { dinner }\end{array}$} \\
\hline & \multicolumn{8}{|c|}{ Percent not meeting recommendations $(95 \% \mathrm{Cl})^{d}$} \\
\hline Saturated fatty acids ${ }^{e}$ & 55.3 & $(52.1-58.5)$ & 57.4 & $(54.0-60.7)$ & 44.2 & $(41.0-47.4)^{* * *}$ & 59.2 & $(56.1-62.4)$ \\
\hline Vitamin $A^{f}$ & 67.5 & $(64.7-70.2)$ & 70.0 & $(67.4-72.7)$ & 63.3 & $(60.5-66.2)$ & 61.3 & $(58.1-64.4)^{* *}$ \\
\hline Vitamin C & 58.7 & $(55.9-61.4)$ & 59.2 & $(56.5-61.9)$ & 58.8 & $(56.0-61.5)$ & 58.9 & $(56.3-61.6)$ \\
\hline Vitamin D & 98.0 & $(97.8-98.2)$ & 98.0 & $(97.8-98.2)$ & 94.0 & $(93.8-94.2)^{* *}$ & 94.0 & $(93.8-94.2)^{* *}$ \\
\hline Vitamin $\mathrm{E}$ & 94.9 & $(93.9-95.8)$ & 95.4 & $(94.4-96.3)$ & 94.1 & $(93.0-95.1)$ & 94.0 & $(92.9-95.0)$ \\
\hline Thiamin & 12.0 & $(10.5-13.6)$ & 12.3 & $(10.7-13.8)$ & 11.5 & $(10.0-13.0)$ & 10.7 & $(9.2-12.2)$ \\
\hline Riboflavin & 12.5 & $(10.9-14.0)$ & 12.8 & $(11.2-14.3)$ & 11.9 & $(10.3-13.4)$ & 11.0 & $(9.5-12.5)$ \\
\hline Niacin & 10.2 & $(8.8-11.6)$ & 10.7 & $(9.3-12.1)$ & 10.6 & $(9.1-12.1)$ & 10.2 & $(8.8-11.6)$ \\
\hline Vitamin $B_{6}$ & 22.6 & $(20.5-24.6)$ & 24.6 & $(22.6-26.7)$ & 22.7 & $(20.8-24.7)$ & 24.8 & $(22.8-26.8)$ \\
\hline Folate $^{g}$ & 30.9 & $(28.4-33.5)$ & 34.8 & $(32.1-37.5)$ & 31.0 & $(28.5-33.6)$ & 29.9 & $(27.4-32.4)$ \\
\hline Vitamin $B_{12}$ & 13.6 & $(12.0-15.3)$ & 13.8 & $(12.1-15.5)$ & 13.4 & $(11.8-15.0)$ & 12.0 & $(10.5-13.5)$ \\
\hline Calcium & 54.7 & $(52.0-57.4)$ & 55.1 & $(52.4-57.9)$ & 55.3 & $(52.6-58.0)$ & 53.6 & $(50.8-56.3)$ \\
\hline Iron & 15.7 & $(14.1-17.4)$ & 15.7 & $(14.1-17.3)$ & 15.7 & $(14.1-17.4)$ & 15.3 & $(13.7-17.0)$ \\
\hline Magnesium & 83.1 & $(81.3-84.9)$ & 84.2 & $(82.4-85.9)$ & 71.8 & $(69.4-74.2)^{* * *}$ & 83.7 & $(82.0-85.4)$ \\
\hline Sodium $^{8}$ & 78.7 & $(76.9-80.5)$ & 78.5 & $(76.7-80.2)$ & 77.8 & (76.0-79.6) & 78.5 & $(76.7-80.3)$ \\
\hline \multirow[t]{2}{*}{ Zinc } & 47.0 & $(44.7-49.3)$ & 49.7 & $(47.3-52.0)$ & 48.0 & $(45.8-50.3)$ & 47.1 & $(44.8-49.4)$ \\
\hline & \multicolumn{8}{|c|}{ Mean intake $(95 \% \mathrm{Cl})$} \\
\hline Energy (kcal) & 2171 & $(2119-2224)$ & 2157 & $(2104-2210)$ & 2162 & $(2110-2215)$ & 2167 & $(2114-2220)$ \\
\hline Protein, total (g) & 81 & $(79-83)$ & 80 & $(78-83)$ & 79 & $(76-81)$ & 75 & $(73-78)^{* *}$ \\
\hline Carbohydrate, total (g) & 267 & $(260-274)$ & 262 & $(255-269)$ & 267 & $(260-274)$ & 270 & $(263-277)$ \\
\hline Fatty acids, total (g) & 81 & $(78-83)$ & 81 & $(78-83)$ & 79 & $(76-81)$ & 78 & $(76-81)$ \\
\hline Fiber $(g)$ & 14 & $(13-14)$ & 14 & $(13-14)$ & 14 & $(13-14)$ & 14 & $(13-14)$ \\
\hline Eicosapentaenoic acid (g) & 0.02 & $(0.02-0.02)$ & 0.02 & $(0.02-0.02)$ & 0.02 & $(0.02-0.02)$ & 0.02 & $(0.02-0.02)$ \\
\hline Docosahexaenoic acid (g) & 0.07 & $(0.06-0.08)$ & 0.07 & $(0.06-0.07)$ & 0.07 & $(0.07-0.08)$ & 0.08 & $(0.07-0.08)$ \\
\hline a-linolenic acid (g) & 1.40 & $(1.35-1.44)$ & 1.40 & $(1.35-1.44)$ & 1.38 & $(1.34-1.43)$ & 1.40 & $(1.35-1.44)$ \\
\hline Vitamin K ( $\mu \mathrm{g})$ & 76 & $(72-79)$ & 76 & $(72-79)$ & 76 & $(72-79)$ & 75 & $(72-79)$ \\
\hline Choline (mg) & 291 & $(282-300)$ & 302 & $(292-312)$ & 324 & $(314-335)^{* * *}$ & 346 & $(334-358)^{* * *}$ \\
\hline Potassium (mg) & 2586 & $(2555-2618)$ & 2569 & $(2539-2599)$ & 2566 & $(2536-2596)$ & 2561 & $(2530-2592)$ \\
\hline a-carotene $(\mu \mathrm{g})$ & 320 & $(292-349)$ & 282 & $(260-304)$ & 283 & $(198-368)$ & 268 & $(156-379)$ \\
\hline$\beta$-carotene $(\mu \mathrm{g})$ & 1417 & $(1331-1504)$ & 1418 & $(1333-1504)$ & 1411 & $(1326-1496)$ & 1389 & $(1306-1472)$ \\
\hline$\beta$-cryptoxanthin $(\mu \mathrm{g})$ & 84 & $(78-90)$ & 77 & $(72-82)$ & 82 & $(76-87)$ & 80 & $(75-85)$ \\
\hline Lutein and zeaxanthin $(\mu \mathrm{g})$ & 998 & (938-1058) & 996 & $(936-1055)$ & 1064 & $(1004-1125)$ & 1110 & $(1046-1175)$ \\
\hline Lycopene $(\mu \mathrm{g})$ & 7514 & $(6764-8264)$ & 7403 & (6717-8088) & 7775 & (7005-8546) & 7578 & (6818-8338) \\
\hline
\end{tabular}

All estimates adjusted for age, gender, race-ethnicity, education, marital status, household size, health insurance, BMI, income-to-poverty ratio, and whether the dietary recall was on a weekday or weekend

${ }^{a}$ Modeled daily nutrient intake if eggs were consumed as the main dish at each eating occasion

${ }^{\mathrm{b}}$ Mean, 2001-2014

${ }^{\mathrm{c}}$ Referent

${ }^{\mathrm{d}}$ Daily nutrient recommendations are Estimated Average Requirements (EAR) unless otherwise specified. National Academy of Sciences, Institute of Medicine. 2006. Dietary reference intakes: The essential guide to nutrient requirements. Jennifer J. Otten, Jennifer Pitzi Hellwig, Linda D. Meyers (eds.). National Academies Press, Washington, DC

eLess than 10\% of total energy. US Department of Health and Human Services \& US Department of Agriculture. 2015-2020. Dietary Guidelines for Americans 2015-2020. U.S. Government Printing Office, Washington, DC. Available at: http://health.gov/dietaryguidelines/ (verified 29 March 2016)

Retinol Activity Equivalent (RAE)

${ }^{9}$ Dietary Folate Equivalent (DFE)

${ }^{\mathrm{h}}$ Tolerable upper intake level limit. National Academy of Sciences, Institute of Medicine. 2006. Dietary reference intakes: The essential guide to nutrient requirements. Jennifer J. Otten, Jennifer Pitzi Hellwig, Linda D. Meyers (eds.). National Academies Press, Washington, DC

*Different than food insecure non-participants at $P<0.017$ (Bonferroni adjusted for multiple comparisons)

**Different than food insecure non-participants at $P<0.003$ (Bonferroni adjusted for multiple comparisons) 
Table 4 Reported daily nutrient intakes and modeled nutrient intakes if eggs were consumed as the main dish at each eating occasion among participants in the Special Supplemental Nutrition Program for Women, Infants, and Children (WIC), $2001-2014$ ( $n=636)$

\begin{tabular}{|c|c|c|c|c|c|c|c|c|}
\hline \multirow[t]{2}{*}{ Nutrient } & \multicolumn{2}{|c|}{ Reported daily intake $e^{b, c}$} & \multicolumn{6}{|c|}{ Modeled daily intake ${ }^{a}$} \\
\hline & & & \multicolumn{2}{|c|}{$\begin{array}{l}\text { Eggs consumed } \\
\text { as main dish at breakfast }\end{array}$} & \multicolumn{2}{|c|}{$\begin{array}{l}\text { Eggs consumed } \\
\text { as main dish at lunch }\end{array}$} & \multicolumn{2}{|c|}{$\begin{array}{l}\text { Eggs consumed } \\
\text { as main dish at dinner }\end{array}$} \\
\hline & \multicolumn{8}{|c|}{ Percent not meeting recommendations $(95 \% \mathrm{Cl})^{d}$} \\
\hline Saturated fatty acids ${ }^{e}$ & 57.5 & $(50.4-64.7)$ & 62.1 & $(55.5-68.7)$ & 40.9 & $(34.1-47.7)^{* *}$ & 63.1 & $(56.5-69.7)$ \\
\hline Vitamin $A^{f}$ & 60.9 & $(53.3-68.6)$ & 66.3 & $(58.8-73.8)$ & 54.5 & $(47.7-61.2)$ & 54.6 & $(46.8-62.5)$ \\
\hline Vitamin C & 47.3 & $(39.2-55.3)$ & 48.9 & $(40.8-57.0)$ & 48.9 & $(40.8-57.0)$ & 47.8 & $(39.9-55.8)$ \\
\hline Vitamin D & 96.0 & $(94.0-98.0)$ & 97.0 & $(96.8-97.2)$ & 95.0 & $(94.0-98.0)$ & 96.0 & $(95.8-96.2)$ \\
\hline Vitamin $\mathrm{E}$ & 97.3 & $(95.9-98.6)$ & 97.6 & $(96.5-98.8)$ & 96.7 & $(95.2-98.3)$ & 96.9 & $(95.4-98.3)$ \\
\hline Thiamin & 15.8 & $(12.6-18.9)$ & 19.0 & $(15.3-22.6)$ & 15.4 & (12.3-18.4) & 14.8 & $(11.6-18.1)$ \\
\hline Riboflavin & 12.7 & $(9.8-15.6)$ & 14.3 & $(11.1-17.4)$ & 11.6 & $(8.9-14.4)$ & 11.1 & $(8.3-13.8)$ \\
\hline Niacin & 9.6 & $(6.9-12.3)$ & 11.6 & $(8.4-14.7)$ & 10.9 & $(7.9-13.8)$ & 9.7 & $(7.0-12.4)$ \\
\hline Vitamin $B_{6}$ & 25.7 & $(20.6-30.9)$ & 33.6 & $(27.3-39.8)$ & 26.4 & $(21.2-31.6)$ & 29.4 & $(23.9-34.9)$ \\
\hline Folate & 27.3 & $(22.3-32.2)$ & 38.9 & $(31.8-46.1)^{* *}$ & 28.0 & $(22.9-33.1)$ & 26.6 & $(21.6-31.6)$ \\
\hline Vitamin $B_{12}$ & 11.3 & $(8.7-13.9)$ & 12.2 & $(9.4-15.1)$ & 10.9 & $(8.4-13.4)$ & 10.0 & $(7.6-12.5)$ \\
\hline Calcium & 44.6 & $(37.8-51.4)$ & 44.92 & $(38.3-51.6)$ & 45.49 & $(38.3-52.7)$ & 42.4 & $(35.8-49.0)$ \\
\hline Iron & 62.6 & $(50.6-74.6)$ & 67.4 & $(56.0-78.9)$ & 62.1 & $(50.1-74.2)$ & 61.9 & $(49.8-73.9)$ \\
\hline Magnesium & 86.9 & $(83.5-90.4)$ & 88.7 & $(85.3-92.0)$ & 63.3 & $(57.1-69.4)^{* * *}$ & 87.5 & $(84.1-90.9)$ \\
\hline Sodium ${ }^{h}$ & 81.4 & $(76.6-86.2)$ & 80.7 & $(75.6-85.8)$ & 80.9 & (75.9-85.9) & 81.2 & $(76.4-85.9)$ \\
\hline \multirow[t]{2}{*}{ Zinc } & 44.2 & $(38.5-49.9)$ & 51.2 & $(45.1-57.2)$ & 46.3 & $(40.1-52.4)$ & 45.5 & $(39.5-51.5)$ \\
\hline & \multicolumn{8}{|c|}{ Mean intake (95\% Cl) } \\
\hline Energy (kcal) & 2309 & $(2202-2416)$ & 2279 & $(2172-2387)$ & 2300 & (2193-2408) & 2304 & (2198-2410) \\
\hline Protein, total (g) & 88 & $(83-92)$ & 88 & $(83-92)$ & 85 & $(80-89)$ & 78 & $(77-79)^{* *}$ \\
\hline Carbohydrate, total (g) & 289 & $(275-304)$ & 256 & $(254-258)^{* *}$ & 289 & $(275-304)$ & 298 & $(283-313)$ \\
\hline Fatty acids, total (g) & 87 & $(81-92)$ & 88 & $(82-93)$ & 85 & $(79-90)$ & 88 & $(82-93)$ \\
\hline Fiber (g) & 14 & $(13-15)$ & 13 & $(13-14)$ & 14 & $(13-15)$ & 14 & $(13-15)$ \\
\hline Eicosapentaenoic acid (g) & 0.02 & $(0.02-0.03)$ & 0.02 & $(0.02-0.03)$ & 0.023 & $(0.02-0.02)$ & 0.019 & $(0.02-0.02)$ \\
\hline Docosahexaenoic acid (g) & 0.07 & $(0.06-0.08)$ & 0.07 & $(0.06-0.08)$ & 0.079 & $(0.06-0.08)$ & 0.069 & $(0.06-0.08)$ \\
\hline a-linolenic acid (g) & 1.38 & $(1.26-1.5)$ & 1.40 & $(1.28-1.51)$ & 1.36 & $(1.24-1.48)$ & 1.41 & $(1.29-1.52)$ \\
\hline Vitamin K ( $\mu \mathrm{g})$ & 72 & $(65-80)$ & 72 & $(65-80)$ & 71 & $(63-78)$ & 71 & $(64-79)$ \\
\hline Choline (mg) & 269 & $(251-287)$ & 286 & $(267-305)$ & 316 & $(294-337)^{* *}$ & 308 & $(285-331)^{* * *}$ \\
\hline Potassium (mg) & 2252 & $(2113-2392)$ & 2233 & $(2092-2373)$ & 2215 & (2070-2360) & 2222 & $(2083-2362)$ \\
\hline a-carotene $(\mu \mathrm{g})$ & 352 & $(274-430)$ & 309 & $(252-367)$ & 307 & $(200-414)$ & 314 & $(174-453)$ \\
\hline$\beta$-carotene $(\mu \mathrm{g})$ & 1395 & $(1187-1603)$ & 1386 & $(1176-1596)$ & 1368 & (1158-1578) & 1390 & $(1181-1599)$ \\
\hline$\beta$-cryptoxanthin ( $\mu \mathrm{g})$ & 102 & $(81-123)$ & 92 & $(75-110)$ & 100 & $(82-118)$ & 96 & $(80-113)$ \\
\hline Lutein and zeaxanthin $(\mu \mathrm{g})$ & 1040 & $(914-1166)$ & 1034 & $(916-1151)$ & 1158 & $(1038-1279)$ & 1141 & $(1013-1268)$ \\
\hline Lycopene ( $\mu \mathrm{g})$ & 7939 & $(6436-9442)$ & 8249 & (6743-9756) & 8078 & (6402-9754) & 9180 & $(7504-10,857)$ \\
\hline
\end{tabular}

All estimates adjusted for age, race-ethnicity, education, marital status, household size, health insurance, BMl, income-to-poverty ratio, and whether the dietary recall was on a weekday or weekend

Modeled daily nutrient intake if eggs were consumed as the main dish at each eating occasion

${ }^{b}$ Mean, 2001-2014

${ }^{3}$ Referent

${ }^{\mathrm{d} D a i l y}$ nutrient recommendations are Estimated Average Requirements (EAR) unless otherwise specified. National Academy of Sciences, Institute of Medicine. 2006. Dietary reference intakes: The essential guide to nutrient requirements. Jennifer J. Otten, Jennifer Pitzi Hellwig, Linda D. Meyers (eds.). National Academies Press, Washington, DC

'Less than 10\% of total energy. US Department of Health and Human Services \& US Department of Agriculture. 2015-2020. Dietary Guidelines for Americans 2015-2020. U.S.

Government Printing Office, Washington, DC. Available at: http://health.gov/dietaryguidelines/ (verified 29 March 2016)

Retinol Activity Equivalent (RAE)

'Dietary Folate Equivalent (DFE)

${ }^{\mathrm{h}}$ Tolerable upper intake level limit. National Academy of Sciences, Institute of Medicine. 2006. Dietary reference intakes: The essential guide to nutrient requirements. Jennifer J. Otten, Jennifer Pitzi Hellwig, Linda D. Meyers (eds.). National Academies Press, Washington, DC

*Different than food insecure non-participants at $P<0.017$ (Bonferroni adjusted for multiple comparisons)

**Different than food insecure non-participants at $P<0.003$ (Bonferroni adjusted for multiple comparisons)

***Different than food insecure non-participants at $P<0.0003$ (Bonferroni adjusted for multiple comparisons) 
Table 5 Reported daily nutrient intakes and modeled nutrient intakes if eggs were consumed as the main dish at each eating occasion among participants in the Supplemental Nutrition Assistance Program (SNAP), 2001-2014 ( $n=4020)$

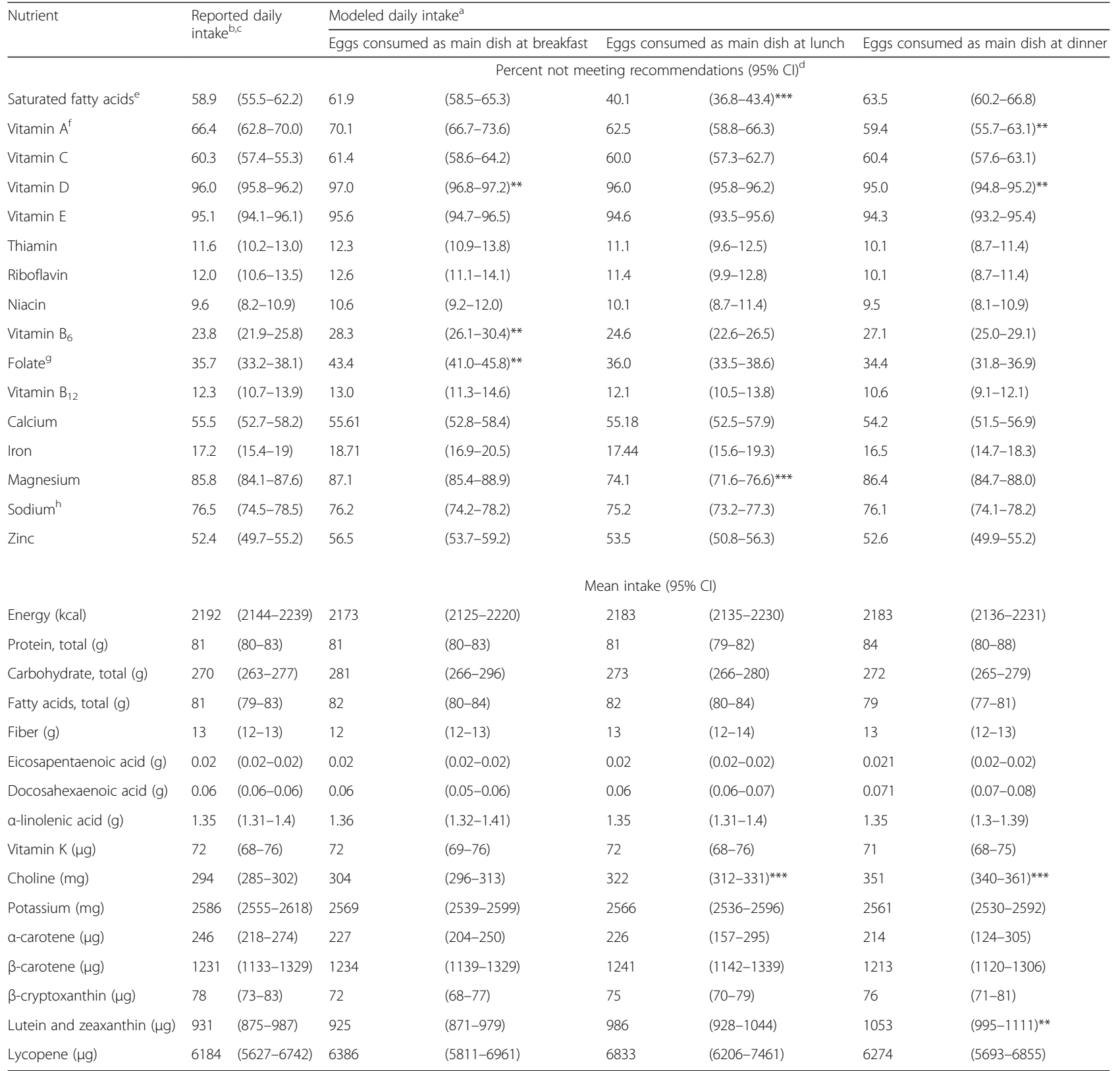

All estimates adjusted for age, gender, race-ethnicity, education, marital status, household size, health insurance, BMI, income-to-poverty ratio, and whether the dietary recall was on a weekday or weekend

a Modeled daily nutrient intake if eggs were consumed as the main dish at each eating occasion

${ }^{b}$ Mean, 2001-2014

'Referent

${ }^{\mathrm{d} D a i l y}$ nutrient recommendations are Estimated Average Requirements (EAR) unless otherwise specified. National Academy of Sciences, Institute of Medicine. 2006.

Dietary reference intakes: The essential guide to nutrient requirements. Jennifer J. Otten, Jennifer Pitzi Hellwig, Linda D. Meyers (eds.). National Academies Press,

Washington, DC

eLess than 10\% of total energy. US Department of Health and Human Services \& US Department of Agriculture. 2015-2020. Dietary Guidelines for Americans

2015-2020. U.S. Government Printing Office, Washington, DC. Available at: http://health.gov/dietaryguidelines/ (verified 29 March 2016)

fRetinol Activity Equivalent (RAE)

fDietary Folate Equivalent (DFE)

'Tolerable upper intake level limit. National Academy of Sciences, Institute of Medicine. 2006. Dietary reference intakes: The essential guide to nutrient requirements.

Jennifer J. Otten, Jennifer Pitzi Hellwig, Linda D. Meyers (eds.). National Academies Press, Washington, DC

*Different than food insecure non-participants at $P<0.017$ (Bonferroni adjusted for multiple comparisons)

**Different than food insecure non-participants at $P<0.003$ (Bonferroni adjusted for multiple comparisons)

****Different than food insecure non-participants at $P<0.0003$ (Bonferroni adjusted for multiple comparisons) 
Table 6 Reported daily nutrient intakes and modeled nutrient intakes if eggs were consumed as the main dish at each eating occasion among food secure non-participants, 2001-2014 ( $n=26,454)$

\begin{tabular}{|c|c|c|c|c|c|c|c|c|}
\hline \multirow[t]{3}{*}{ Nutrient } & \multirow{2}{*}{\multicolumn{2}{|c|}{$\begin{array}{l}\text { Reported daily } \\
\text { intake }\end{array}$}} & \multicolumn{6}{|c|}{ Modeled daily intake ${ }^{a}$} \\
\hline & & & \multicolumn{2}{|c|}{ Eggs consumed as main dish at breakfast } & \multicolumn{2}{|c|}{ Eggs consumed as main dish at lunch } & \multicolumn{2}{|c|}{ Eggs consumed as main dish at dinne } \\
\hline & \multicolumn{8}{|c|}{ Percent not meeting recommendations $(95 \% \mathrm{Cl})^{d}$} \\
\hline Saturated fatty acids ${ }^{\mathrm{e}}$ & 62.1 & $(60.6-63.6)$ & 65.6 & $(64.2-67.1)^{*}$ & 37.0 & $(35.4-38.5)^{* * *}$ & 66.3 & $(64.8-67.8)^{* *}$ \\
\hline Vitamin $A^{f}$ & 46.9 & $(45.4-48.4)$ & 50.1 & $(48.6-51.6)^{* *}$ & 42.7 & $(41.2-44.2)^{* * *}$ & 40.4 & $(38.9-41.9)^{* *}$ \\
\hline Vitamin C & 45.9 & $(44.3-47.5)$ & 47.2 & $(45.6-48.8)$ & 46.1 & $(44.5-47.7)$ & 46.4 & $(44.8-48.0)$ \\
\hline Vitamin D & 95.0 & $(94.8-95.2)$ & 95.0 & $(94.8-95.2)$ & 94.0 & $(93.8-94.2)^{* *}$ & 94.0 & $(93.8-94.2)^{* *}$ \\
\hline Vitamin E & 88.1 & $(87.0-89.2)$ & 89.7 & $(88.7-90.6)$ & 87.0 & $(85.9-88.1)$ & 86.5 & $(85.4-87.6)$ \\
\hline Thiamin & 9.5 & $(8.8-10.2)$ & 9.7 & $(9.0-10.5)$ & 9.3 & $(8.5-10.0)$ & 8.9 & $(8.1-9.6)$ \\
\hline Riboflavin & 9.7 & $(9.0-10.4)$ & 9.9 & $(9.2-10.7)$ & 9.4 & $(8.6-10.1)$ & 8.9 & $(8.2-9.6)$ \\
\hline Niacin & 9.3 & $(8.5-10.0)$ & 10.2 & $(9.4-10.9)$ & 9.6 & $(8.8-10.3)$ & 9.3 & $(8.5-10.0)$ \\
\hline Vitamin $B_{6}$ & 18.8 & $(17.6-19.9)$ & 22.6 & $(21.3-23.9)^{* * *}$ & 19.1 & $(18.0-20.3)$ & 20.8 & $(19.6-21.9)$ \\
\hline Folate $^{j}$ & 27.9 & $(26.8-29.0)$ & 35.8 & $(34.6-37.0)^{* *}$ & 27.9 & $(26.7-29.0)$ & 27.1 & $(25.9-28.2)$ \\
\hline Vitamin $B_{12}$ & 10.8 & $(10.0-11.6)$ & 11.4 & $(10.5-12.3)$ & 10.6 & $(9.8-11.4)$ & 9.9 & $(9.1-10.6)$ \\
\hline Calcium & 47.4 & $(46.2-48.6)$ & 48.32 & $(47.1-49.5)$ & 47.55 & $(46.4-48.7)$ & 46.3 & $(45.1-47.5)$ \\
\hline Iron & 12.7 & $(11.9-13.5)$ & 13.62 & $(12.8-14.4)$ & 12.61 & $(11.8-13.4)$ & 12.3 & $(11.6-13.1)$ \\
\hline Magnesium & 72.1 & $(70.9-73.3)$ & 74.7 & $(73.5-75.8)^{*}$ & 58.8 & $(57.3-60.3)^{* * *}$ & 73.1 & $(71.9-74.3)$ \\
\hline Sodium ${ }^{\mathrm{h}}$ & 81.4 & $(80.5-82.3)$ & 81.1 & $(80.2-82.0)$ & 80.5 & $(79.6-81.4)$ & 81.3 & $(80.5-82.2)$ \\
\hline \multirow[t]{2}{*}{ Zinc } & 39.4 & $(38.2-40.6)$ & 44.0 & $(42.8-45.3)^{* * *}$ & 40.1 & $(38.9-41.3)$ & 39.5 & $(38.2-40.7)$ \\
\hline & \multicolumn{8}{|c|}{ Mean intake (95\% Cl) } \\
\hline Energy (kcal) & 2176 & $(2159-2192)$ & 2158 & $(2142-2175)$ & 2170 & $(2153-2186)$ & 2171 & $(2154-2187)$ \\
\hline Protein, total (g) & 84 & $(83-84)$ & 83 & $(82-84)$ & 81 & $(81-82)^{* * *}$ & 76 & $(74-78)^{* * *}$ \\
\hline Carbohydrate, total (g) & 262 & $(260-264)$ & 264 & $(258-271)$ & 262 & $(260-264)$ & 265 & $(262-267)$ \\
\hline Fatty acids, total (g) & 83 & $(82-84)$ & 83 & $(82-83)$ & 81 & $(80-82)^{*}$ & 80 & $(79-81)$ \\
\hline Fiber (g) & 16 & $(16-16)$ & 15 & $(15-15)^{* * *}$ & 16 & $(15-16)$ & 16 & $(15-16)$ \\
\hline Eicosapentaenoic acid (g) & 0.03 & $(0.03-0.03)$ & 0.03 & $(0.03-0.03)$ & 0.03 & $(0.02-0.03)$ & 0.03 & $(0.02-0.03)$ \\
\hline Docosahexaenoic acid (g) & 0.08 & $(0.08-0.08)$ & 0.08 & $(0.08-0.08)$ & 0.08 & $(0.08-0.09)$ & 0.09 & $(0.09-0.09)$ \\
\hline a-linolenic acid (g) & 1.53 & $(1.50-1.55)$ & 1.53 & $(1.51-1.55)$ & 1.52 & $(1.50-1.54)$ & 1.53 & $(1.50-1.55)$ \\
\hline Vitamin $\mathrm{K}(\mu \mathrm{g})$ & 100 & $(97-103)$ & 100 & $(97-103)$ & 100 & $(97-103)$ & 99 & $(96-102)$ \\
\hline Choline (mg) & 317 & $(312-322)$ & 336 & $(331-341)$ & 353 & $(348-358)^{* * *}$ & 382 & $(376-388)^{* * *}$ \\
\hline Potassium (mg) & 2222 & $(2159-2286)$ & 2219 & $(2158-2279)$ & 2207 & $(2145-2269)$ & 2205 & $(2143-2267)$ \\
\hline a-carotene $(\mu \mathrm{g})$ & 516 & $(488-544)$ & 426 & $(405-446)$ & 425 & $(300-551)$ & 403 & $(236-570)$ \\
\hline$\beta$-carotene $(\mu \mathrm{g})$ & 2136 & (2054-2218) & 2134 & (2053-2214) & 2138 & $(2057-2219)$ & 2104 & $(2022-2186)$ \\
\hline$\beta$-cryptoxanthin $(\mu \mathrm{g})$ & 112 & $(108-116)$ & 103 & $(99-107)$ & 107 & $(103-111)$ & 105 & $(101-109)$ \\
\hline Lutein and zeaxanthin $(\mu \mathrm{g})$ & 1418 & $(1362-1473)$ & 1436 & $(1383-1490)$ & 1489 & $(1435-1543)$ & 1544 & $(1491-1598)^{* *}$ \\
\hline Lycopene $(\mu \mathrm{g})$ & 8218 & (7838-8598) & 8096 & (7759-8433) & 8653 & $(8242-9065)$ & 8290 & (7920-8660) \\
\hline
\end{tabular}

All estimates adjusted for age, gender, race-ethnicity, education, marital status, household size, health insurance, BMI, income-to-poverty ratio, and whether the dietary recall was on a weekday or weekend

${ }^{a}$ Modeled daily nutrient intake if eggs were consumed as the main dish at each eating occasion

${ }^{b}$ Mean, 2001-2014

'Referent

${ }^{\mathrm{d} D a i l y}$ nutrient recommendations are Estimated Average Requirements (EAR) unless otherwise specified. National Academy of Sciences, Institute of Medicine. 2006.

Dietary reference intakes: The essential guide to nutrient requirements. Jennifer J. Otten, Jennifer Pitzi Hellwig, Linda D. Meyers (eds.). National Academies Press,

Washington, DC

eLess than 10\% of total energy. US Department of Health and Human Services \& US Department of Agriculture. 2015-2020. Dietary Guidelines for Americans

2015-2020. U.S. Government Printing Office, Washington, DC. Available at: http://health.gov/dietaryguidelines/ (verified 29 March 2016)

fRetinol Activity Equivalent (RAE)

'Dietary Folate Equivalent (DFE)

${ }^{\mathrm{h}}$ Tolerable upper intake level limit. National Academy of Sciences, Institute of Medicine. 2006. Dietary reference intakes: The essential guide to nutrient requirements.

Jennifer J. Otten, Jennifer Pitzi Hellwig, Linda D. Meyers (eds.). National Academies Press, Washington, DC

*Different than food insecure non-participants at $P<0.017$ (Bonferroni adjusted for multiple comparisons)

**Different than food insecure non-participants at $P<0.003$ (Bonferroni adjusted for multiple comparisons)

****Different than food insecure non-participants at $P<0.0003$ (Bonferroni adjusted for multiple comparisons) 
$(P<0.001)$. No changes in nutrient intake were observed when eggs were substituted at dinner, except a decrease in mean intake of protein $(P<0.001)$ and an increase in mean intake of choline $(P<0.01)$. Among SNAP participants (Table 5), substituting eggs at breakfast increased the percent not meeting recommendations for vitamin $\mathrm{D}$ $(P<0.01)$, vitamin $\mathrm{B}_{6}(P<0.01)$, and folate $(P<0.01)$, and substituting eggs at lunch decreased the percent not meeting recommendations for saturated fatty acids $(P<0.001)$ and magnesium $(P<0.001)$. Substituting eggs at dinner decreased the percent not meeting recommendations for vitamin $\mathrm{A}(P<0.01)$ and vitamin $\mathrm{D}(P<0.01)$. Among food secure non-participants (Table 6), substituting eggs at breakfast increased the percent of individuals not meeting recommendations for saturated fatty acids $(P<0.05)$, vitamin A $(P<0.01)$, vitamin $\mathrm{B}_{6}(P<0.001)$, folate $(P<0.01)$, magnesium $(P<0.05)$, and zinc $(P<0.001)$, and decreased the mean intake of fiber $(\mathrm{P}<0.001)$. Substituting eggs at lunch decreased the percent not meeting recommendations for saturated fatty acids $(P<0.001)$, vitamin A $(P<0.001)$, vitamin $\mathrm{D}(P<0.01)$, and magnesium $(P<0.001)$, and decreased the mean intake of protein and total fatty acids $(\mathrm{P}<0.001)$. Substituting eggs at dinner increase the percent not meeting recommendations for saturated fatty acids $(P<0.001)$, but decreased the percent not meeting recommendations for vitamins $\mathrm{A}$ and $\mathrm{D}(P<0.01)$.

\section{Total nutrient index}

Figure 1a-d display the results of the total nutrient index, which represents the percent change from the reported intake of all 31 nutrients collectively when eggs are substituted for the most commonly consumed main dish at each eating occasion. Among each group (i.e., food insecure non-participants, SNAP participants, WIC participants, and food secure non-participants), total nutrient intake did not change at breakfast, lunch, or dinner $(P>0.05)$. Sensitivity analyses demonstrated that individual nutrients have minimal effect on the reliability of the total nutrient index (Additional file 7: Table S7).

\section{Discussion}

In this nationally representative sample of nearly 35,000 adults surveyed over 14 years, meaningful disparities in daily intake of several key nutrients were observed between distinct populations differentiated by food security status and participation in federal food assistance programs. Importantly, using dietary modeling techniques, we demonstrated that well-defined, moderate dietary changes at the population-level can increase intake of some, but not all, nutrients, and can reduce the prevalence of nutrient inadequacy among these nutritionally vulnerable populations.
Diet modeling has become a popular tool for estimating the impact of food substitutions on nutrient adequacy at the population level $[18,19,23,38]$. Yet to the best of our knowledge, the present study is the first to use diet modeling to examine the effects of food substitutions at multiple eating occasions on meeting daily nutrient intake recommendations among distinct groups of nutritionally vulnerable populations.

Consistent with our findings, a review by Andreyeva et al. [9] indicated lower nutrient intake among SNAP participants compared to higher income non-participants. Leung et al. [39] analyzed NHANES (1998-2008) dietary data from 918 SNAP participants and reported that 52 and $67 \%$ did not meet recommendations for folate and calcium, respectively, which are higher than our estimates (36\% for folate and $56 \%$ for calcium). These disagreements may stem from differences in sample size ( $n=3724$ SNAP participants in the present study) resulting from different inclusion criteria. In contrast to Leung et al., [39] we categorized SNAP participants regardless of age or income, which allowed for greater sample size and improved generalizability to the target population. To the best of our knowledge, no previous studies have examined nutrient adequacy among adult WIC participants, although several studies have observed an overall improvement in the healthfulness of food choices among WIC participants since $2009[8,40]$. Our results for the proportion of food insecure non-participants meeting individual nutrient recommendations are largely consistent with others [41].

In the present study, we demonstrated that consuming eggs as the main dish at lunch or dinner did not have an effect on total nutrient intake among nutritionally vulnerable populations (i.e., food insecure non-participants, WIC participants, and SNAP participants) or their food secure nonparticipant counterparts. However, our findings for several specific nutrients warrant particular mention. Importantly, we observed a meaningful reduction in the prevalence of food insecure non-participants not meeting daily vitamin $\mathrm{D}$ recommendations when eggs were consumed as the main dish at lunch or dinner. The 2015-2020 Dietary Guidelines for Americans proclaimed vitamin D a nutrient of public health concern [42] given the strikingly high prevalence of intake inadequacy (92-97\%) [43] and its relationship to chronic disease patterns [42]. Yet, when eggs were consumed as the main dish at breakfast, the prevalence of folate inadequacy increased for WIC participants and SNAP participants, which is concerning. Folate is a critical nutrient for fetal neural tube development, which is the reason for elevated intake recommendations during pregnancy [44]. This is of particular concern for adult WIC participants, over $40 \%$ of whom are pregnant and, of those, over half enrolled during their first trimester [4] when adequate folate intake is most critical [45]. We estimated that breakfast cereal, much 


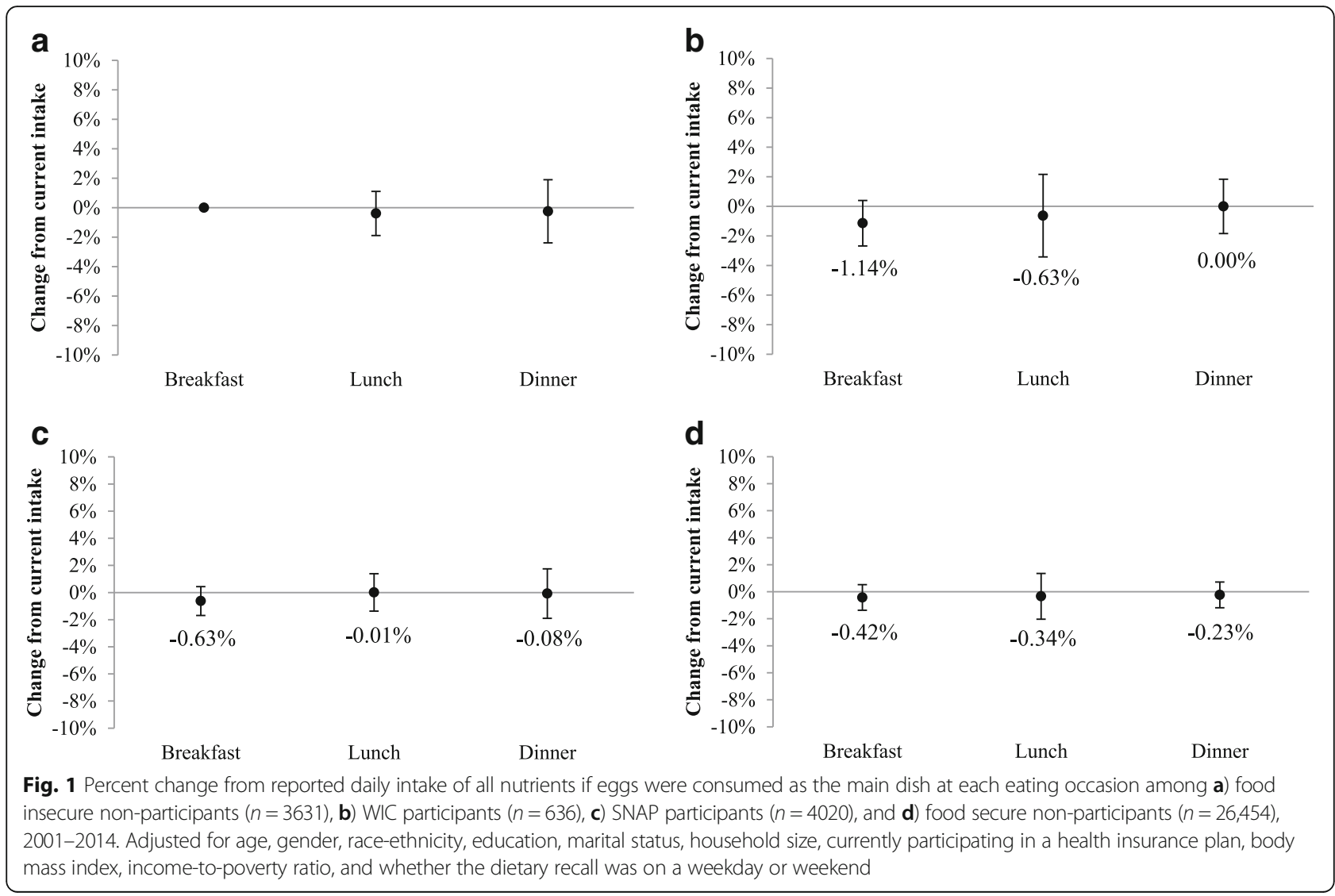

of which is fortified with folic acid, was the most commonly consumed main dish at breakfast among all groups, so it is not surprising that consuming eggs instead of breakfast cereal resulted in reduced folate (i.e. dietary folate equivalent) intake. Although others have recently reported successful innovations in the development of folate-enriched eggs, [46] more research is needed before these products can be successfully introduced to consumers on a large scale.

Several important strengths of this study warrant mention. To the best of our knowledge this is the first study to assess nutrient intake among distinct nutritionally vulnerable populations simultaneously, for a diverse array of 31 nutrients including macronutrients, vitamins, minerals, and carotenoids. NCI methods and macros were used to estimate usual intake distributions, which represent the gold standard for addressing the foremost statistical challenges when assessing nutrient intake among populations [47]. We also used a novel approach, the total nutrient index, to assess intake of all 31 nutrients collectively, which accounts for relative differences in the standard errors of each nutrient. Finally, and importantly, the large sample size $(n>34,000)$ and national representation of our data make our findings generalizable to each of our study populations.

This study is also not without limitations. We conducted our food substitution analyses on a gram basis, ensuring that equal grams of dishes were being substituted; other approaches include using a serving basis or calorie basis. In the present study our data represented main dishes as they were reported consumed, which included a combination of individual foods with distinct serving sizes, thus it was not possible to use the serving basis for this analysis. We tested using the calorie basis for food substitutions and observed that it provided essentially the same results as the gram basis ( $\sim 1 \%$ difference in calories between food substitutions). It is also possible that pooling data across NHANES waves may have masked changes in nutrient intake among some groups, particularly WIC participants [8, 40]. Yet it was necessary to pool these data, especially for WIC participants, due to small sample sizes in individual waves, in order to observe clinically and statistically meaningful results.

Regular assessment of nutrient inadequacy at the population level is a crucial component of the public health nutrition agenda in the US, and nutritional monitoring of low income and other vulnerable populations is of particular importance. On the one hand, it is reassuring that populations with a high prevalence of nutrient inadequacy for key nutrients are participating in federal food assistance programs. On the other hand, more research is needed to better understand why many incomeeligible food insecure individuals do not participate in these 
vital programs (over 50\% in this study), given their high prevalence of nutrient inadequacy. Continued efforts are needed to ensure that nutritionally vulnerable populations have access to healthy foods, and that at-risk individuals are counseled to make food choices that promote nutrient adequacy. Additional research is needed to better understand how food substitutions affect diet costs, which may be an important driver of food purchasing decisions among low income individuals with limited food budgets $[6,7]$.

\section{Conclusions}

This study is the first to use diet modeling to examine the effects of food substitutions at multiple eating occasions on meeting daily nutrient intake recommendations among distinct groups of nutritionally vulnerable populations. A novel total nutrient index was also used to estimate changes in the intake of a broad array of diverse nutrients collectively. Regular assessment of nutrient inadequacy at the population level is a crucial component of the public health nutrition agenda in the US, and nutritional monitoring of low income and other vulnerable populations is of particular importance. Clinically meaningful disparities in nutrient intake remain between distinct populations differentiated by food security status and participation in federal food assistance programs. Well-defined and moderate dietary changes at the population-level have the potential to increase nutrient intake and reduce the prevalence of nutrient inadequacy among these nutritionally vulnerable populations, particularly at lunch and dinner. However, careful consideration should be awarded to food substitutions at breakfast, particularly among individuals who are pregnant or trying to become pregnant, in order to maintain adequate folate status. Further research is needed in two key areas: to better understand why many income-eligible food insecure individuals do not participate in federal food assistance programs, and to better understand how food substitutions affect diet costs among low income individuals with limited food budgets.

\section{Additional files}

Additional file 1: Table S1. Foods included in main dish groups. (DOCX $21 \mathrm{~kb})$

Additional file 2: Table S2. Daily nutrient intake recommendations. (DOCX $26 \mathrm{~kb}$ )

Additional file 3: Table S3. Percent of individuals consuming types of main dishes at each eating occasion, 2001-2014 ( $n=34,741)$. (DOCX 23 kb)

Additional file 4: Table S4. Percent of individuals consuming types of egg dishes at each eating occasion, 2001-2014 ( $n=34,741)$. (DOCX $17 \mathrm{~kb})$

Additional file 5: Table S5. Amount of each dish used in substitution modeling. (DOCX $21 \mathrm{~kb}$ )
Additional file 6: Table S6. Nutrient content of dishes used in substitution modeling. (DOCX $32 \mathrm{~kb}$ )

Additional file 7: Table S7. Sensitivity analysis for total nutrient index. (DOCX $27 \mathrm{~kb}$ )

\section{Abbreviations}

24HR: 24-h recall; DGA: Dietary guidelines for Americans; FNDDS: Food and nutrient database for dietary studies; NCl: National Cancer Institute; NHANES: National Health and Nutrition Examination Survey; SNAP: Supplemental Nutrition Assistance Program; USDA: United States Department of Agriculture; WIC: Special Supplemental Nutrition Program for Women, Infants, and Children; WWEIA: What we eat in America

\section{Funding}

This research was funded by the Egg Nutrition Center of the American Egg Board. The funding body did not have any role in the design of the study, data collection, data analysis, and interpretation of the data, and in writing the manuscript. Partial funding was also received from USDA-ARS 3062-51000-051-00D

\section{Availability of data and materials}

The datasets used in this study are publically available at https://wwwn.cdc.gov/ nchs/nhanes/Default.aspx.

\section{Authors' contributions}

$Z C$, JNR, and $L$ designed the research; ZC conducted the research; ZC and LKJ provided essential materials; ZC, LKJ, and WJY analyzed the data and performed statistical analyses; ZC wrote the paper; ZC and LJ had primary responsibility for final content; all authors have read and approved the final manuscript.

\section{Ethics approval and consent to participate}

This study uses publically available secondary data, and was deemed exempt by the University of North Dakota Institutional Review Board.

\section{Competing interests}

The authors declare that they have no competing interests.

\section{Publisher's Note}

Springer Nature remains neutral with regard to jurisdictional claims in published maps and institutional affiliations.

\section{Author details}

${ }^{1}$ Grand Forks Human Nutrition Research Center, US Department of Agriculture, Agricultural Research Service, 2420 2nd Ave. N, Grand Forks, ND 58203, USA. ${ }^{2}$ Center for Food Safety and Applied Nutrition, US Department of Health and Human Services, Food and Drug Administration, 5001 Campus Drive, College Park, MD 20740, USA.

Received: 13 September 2017 Accepted: 1 May 2018

Published online: 17 May 2018

References

1. Supplemental Nutrition Assistance Program (SNAP) eligibility [Available at: http://www.fns.usda.gov/snap/eligibility (verified 16 June 2017)].

2. US Department of Agriculture, Food and Nutrition Service: Supplemental Nutrition Assistance Program (SNAP) national level annual summary. 2016.

3. US Department of Agriculture: FY 2016 Budget Summary and Performance Plan. 2015.

4. US Department of Agriculture, Food and Nutrition Service: WIC Participant and Program Characteristics Final Report. 2015.

5. U S Burden of Disease Collaborators. The state of U.S. health, 1990-2010: burden of diseases, injuries, and risk factors. JAMA. 2013;310:591-606.

6. Kirkpatrick SI, Dodd KW, Reedy J, Krebs-Smith SM. Income and Race/ Ethnicity Are Associated with Adherence to Food-Based Dietary Guidance among US Adults and Children. J Acad Nutr Diet. 2012;112:624-35.

7. Hiza HAB, Casavale KO, Guenther PM, Davis CA. Diet Quality of Americans Differs by Age, Sex, Race/Ethnicity, Income, and Education Level. J Acad Nutr Diet. 2013;113:297-306. 
8. Tester JM, Leung CW, Crawford PB. Revised WIC Food Package and Children's Diet Quality. Pediatrics. 2016;137

9. Andreyeva T, Tripp AS, Schwartz MB. Dietary quality of Americans by Supplemental Nutrition Assistance Program participation status: a systematic review. Am J Prev Med. 2015;49:594-604.

10. Conrad Z, Rehm CD, Wilde P, Mozaffarian D. Cardiometabolic Mortality by Supplemental Nutrition Assistance Program Participation and Eligibility in the United States. Am J Public Health. 2017:e1-9.

11. Damschroder L, Lutes LD, Kirsh S, Kim HM, Gillon L, Holleman RG, Goodrich DE, Lowery JC, Richardson CR. Small-Changes Obesity Treatment Among Veterans: 12-Month Outcomes. Am J Prev Med. 2014;47:541-53.

12. Lutes LD, DiNatale E, Goodrich DE, Ronis DL, Gillon L, Kirsh S, Richardson CR, Damschroder LJ. A randomized trial of a small changes approach for weight loss in veterans: Design, rationale, and baseline characteristics of the ASPIREVA trial. Contemp Clin Trials. 2013:34:161-72.

13. Hills AP, Byrne NM, Lindstrom R, Hill JO. 'Small changes' to diet and physical activity behaviors for weight management. Obesity Facts. 2013;6:228-38.

14. de Castro JM: The time of day of food intake influences overall intake in humans. J Nutr 2004, 134:104-111.

15. Howarth NC, Huang TT, Roberts SB, Lin BH, McCrory MA. Eating patterns and dietary composition in relation to BMI in younger and older adults. Int J Obesity. 2007;31:675-84.

16. Berner $L A$, Becker $G$, Wise M, Doi J. Characterization of dietary protein among older adults in the United States: amount, animal sources, and meal patterns. J Acad Nutr Diet. 2013;113:809-15.

17. Nicklas TA, O'Neil CE, Zanovec M, Keast DR, Fulgoni VL 3rd. Contribution of beef consumption to nutrient intake, diet quality, and food patterns in the diets of the US population. Meat Sci. 2012;90:152-8.

18. Quann EE, Fulgoni VL 3rd, Auestad N. Consuming the daily recommended amounts of dairy products would reduce the prevalence of inadequate micronutrient intakes in the United States: diet modeling study based on NHANES 2007-2010. Nutr J. 2015;14:90.

19. Fulgoni VL III, Keast DR, Auestad N, Quann EE. Nutrients from dairy foods are difficult to replace in diets of Americans: Food pattern modeling and an analyses of the National Health and Nutrition Examination Survey 2003-2006. Nutr Res. 2011:31:759-65.

20. Nicklas TA, O'Neil CE, Fulgoni VL 3rd. The nutritional role of flavored and white milk in the diets of children. J Sch Health. 2013;83:728-33.

21. Fulgoni VL, Buckley RB. The Contribution of Fortified Ready-to-Eat Cereal to Vitamin and Mineral Intake in the U.S. Population, NHANES 2007-2010. Nutrients. 2015;7:3949-58.

22. O'Neil CE, Nicklas TA, Rampersaud GC, Fulgoni VL 3rd. 100\% orange juice consumption is associated with better diet quality, improved nutrient adequacy, decreased risk for obesity, and improved biomarkers of health in adults: National Health and Nutrition Examination Survey, 2003-2006. Nutr J. 2012;11:107.

23. Nicklas TA, Liu Y, Islam N, O'Neil CE. Removing Potatoes from Children's Diets May Compromise Potassium Intake. Adv Nutr. 2016;7:247s-53s

24. Fulgoni VL 3rd, Dreher M, Davenport AJ. Avocado consumption is associated with better diet quality and nutrient intake, and lower metabolic syndrome risk in US adults: results from the National Health and Nutrition Examination Survey (NHANES) 2001-2008. Nutr J. 2013:12:1.

25. Freedman MR, Fulgoni VL 3rd. Canned Vegetable and Fruit Consumption Is Associated with Changes in Nutrient Intake and Higher Diet Quality in Children and Adults: National Health and Nutrition Examination Survey 2001-2010. J Acad Nutr Diet. 2016:116:940-8.

26. Griffin BA. Eggs: good or bad? Proc Nutr Soc. 2016;75:259-64.

27. US Department of Agriculture, Agricultural Research Service: National Nutrient Database for Standard Reference, Release 28. Nutrient Data Laboratory. 2016.

28. Abdel-Aal EM, Akhtar H, Zaheer K, Ali R. Dietary Sources of Lutein and Zeaxanthin Carotenoids and Their Role in Eye Health. Nutrients. 2013;5:1169-85.

29. Kim JE, Gordon SL, Ferruzzi MG, Campbell WW. Effects of egg consumption on carotenoid absorption from co-consumed, raw vegetables. Am J Clin Nutr. 2015;102:75-83.

30. Kim JE, Ferruzzi MG, Campbell WW. Egg Consumption Increases Vitamin E Absorption from Co-Consumed Raw Mixed Vegetables in Healthy Young Men. J Nutr. 2016;146:2199-205.

31. Miranda JM, Anton X, Redondo-Valbuena C, Roca-Saavedra P, Rodriguez JA, Lamas A, Franco CM, Cepeda A. Egg and egg-derived foods: effects on human health and use as functional foods. Nutrients. 2015:7:706-29.
32. Lewis ED, Subhan FB, Bell RC, McCargar LJ, Curtis JM, Jacobs RL, Field CJ. Estimation of choline intake from $24 \mathrm{~h}$ dietary intake recalls and contribution of egg and milk consumption to intake among pregnant and lactating women in Alberta. Br J Nutr. 2014;112:112-21.

33. US Department of Health and Human Services, Centers for Disease Control and Prevention (CDC): About the National Health and Nutrition Examination Survey. 2017.

34. Moshfegh AJ, Rhodes DG, Baer DJ, Murayi T, Clemens JC, Rumpler WV, Paul DR, Sebastian RS, Kuczynski KJ, Ingwersen LA, et al. The US Department of Agriculture Automated Multiple-Pass Method reduces bias in the collection of energy intakes. Am J Clin Nutr. 2008;88:324-32.

35. Bickel G, Nord M, Price C, Hamilton W, Cook J. Guide to measuring household food security: US Department of Agriculture; 2000.

36. US Department of Agriculture, Agricultural Research Service. Food and Nutrient Database for Dietary Studies. 2001-2014.

37. Usual Dietary Intakes: SAS Macros for Analysis of a Single Dietary Component, version 2.1 [Available at: http://epi.grants.cancer.gov/diet/usualintakes/macros_ single.html\#ver2 (verified 16 June 2017)].

38. Houchins JA, Cifelli CJ, Demmer E, Fulgoni lii VL. Diet Modeling in Older Americans: The Impact of Increasing Plant-Based Foods or Dairy Products on Protein Intake. J Nutr Health Aging. 2017;21:673-80.

39. Leung CW, Ding EL, Catalano PJ, Villamor E, Rimm EB, Willett WC. Dietary intake and dietary quality of low-income adults in the Supplemental Nutrition Assistance Program. Am J Clin Nutr. 2012;96:977-88.

40. Schultz DJ, Byker Shanks C, Houghtaling B. The Impact of the 2009 Special Supplemental Nutrition Program for Women, Infants, and Children Food Package Revisions on Participants: A Systematic Review. J Acad Nutr Diet. 2015;115:1832-46.

41. Leung CW, Epel ES, Ritchie LD, Crawford PB, Laraia BA. Food Insecurity Is Inversely Associated with Diet Quality of Lower-Income Adults. J Acad Nutr Diet. 2014;114:1943-U1329.

42. US Department of Health and Human Services and US Department of Agriculture. Dietary Guidelines for Americans 2015-2020, Chapter 2. 8th ed. edition. Washington, DC2015-2020.

43. Dietary Guidelines Advisory Committee. Scientific Report of the 2015 Dietary Guidelines Advisory Committee: Advisory Report to the Secretary of Health and Human Services and the Secretary of Agriculture. Appendix E-2. 1. Washington, DC2015.

44. National Academy of Sciences, Institute of Medicine. Dietary Reference Intakes: The Essential Guide to Nutrient Requirements. Washington, DC: Jennifer J. Otten, Jennifer Pitzi Hellwig, Linda D. Meyers (eds.); 2006.

45. McNulty B, McNulty H, Marshall B, Ward M, Molloy AM, Scott JM, Dornan J, Pentieva K. Impact of continuing folic acid after the first trimester of pregnancy: findings of a randomized trial of Folic Acid Supplementation in the Second and Third Trimesters. Am J Clin Nutr. 2013:98:92-8.

46. Altic L, McNulty H, Hoey L, McAnena L, Pentieva K. Validation of Folate-Enriched Eggs as a Functional Food for Improving Folate Intake in Consumers. Nutrients. 2016;8

47. Tooze JA, Midthune D, Dodd KW, Freedman LS, Krebs-Smith SM, Subar AF, Guenther PM, Carroll RJ, Kipnis V. A new statistical method for estimating the usual intake of episodically consumed foods with application to their distribution. J Am Diet Assoc. 2006;106:1575-87.

\section{Ready to submit your research? Choose BMC and benefit from:}

- fast, convenient online submission

- thorough peer review by experienced researchers in your field

- rapid publication on acceptance

- support for research data, including large and complex data types

- gold Open Access which fosters wider collaboration and increased citations

- maximum visibility for your research: over $100 \mathrm{M}$ website views per year

At BMC, research is always in progress.

Learn more biomedcentral.com/submissions 